\title{
Política Sanitaria y Legislación en Materia de Medicina Indígena Tradicional en México
}

\author{
Health Policy and Legislation Concerning Traditional Indigenous \\ Medicine in México
}

Jaime Tomás P. Page ${ }^{1}$

\section{PAGE, J. T. P. Health Policy and Legislation Concerning Traditional Indigenous Medicine in}

Mexico. Cad. Saúde Públ., Rio de Janeiro, 11 (2): 201-211, Apr/Jun, 1995.

Various Mexican federal and state government agencies dealing with indigenous affairs are trying to establish control over traditional medicine and by recognizing it achieve cost decreases for health care directed towards Indian groups, bolstering it as a first-care level. The result is that the community absorbs the costs of medical care, leading to a reduction in the number of patients seeking government-run health care services. Traditional indigenous healers and their organizations consider the law a two-edged sword. On the one hand it provides them with legal measures for their protection, but on the other, they perceive it as a dangerous threat to their culture and practices. This has led them to seek legal advice, training, and active participation in the drafting process for such legislation. The result of this project is that they have been able to learn about the law, discuss their demands, and work out a proposal which is included in this document.

Key words: Ameridian Health; Health Policy; Health Systems; Health Legislation; Traditional Indigenous Medicine

\section{INTRODUCCION}

Este trabajo contiene resultados parciales del proyecto de investigación denominado "Opiniones y propuestas de dos organizaciones de médicos indígenas tradicionales en torno al proceso de legislación en materia de medicina tradicional". Proyecto que se realiza mediante convenio firmado entre la Organización de Médicos Tradicionales del Estado de Chiapas A. C. (OMIECH), la Organización de Terapeutas Indígenas Tzeltales A. C. (ODETIT), y el Instituto de Estudios Indígenas de la Universidad Autónoma de Chiapas. La iniciativa para su implementación proviene de una propuesta hecha por las organizaciones de médicos

${ }^{\prime}$ Instituto de Estudios Indígenas, Universidad Autónoma de Chiapas. Calle Felipe Flores No. 14., Centro, CP 29200, San Cristóbal de las Casas, Chiapas, México. indígenas tradicionales antes mencionadas, sobre la basede la necesidad que tienen de formarse un criterio respecto a la legalidad mexicana y de sistematizar las opiniones y propuestas surgidas en el seno de las comunidades sociais en torno a la legislación de los derechos de los pueblos indios y específicamente sobre la legislación en torno a la medicina indígena tradicional.

La inquietud para realizar dicho proyecto surge a partir de que el gobierno del estado de Chiapas emite una ley encaminada al control de los médicos indígenas tradicionales y sus prácticas, misma que quedó documentada en el "Capítulo IV de la Ley de Salud del Estado" (Congreso del Estado de Chiapas, 1990). Y, por otra parte, a partir de que el gobierno Federal los invita a participar en diferentes foros (Congreso Nacional de Médicos Indígenas, 1989; I Congreso Nacional de Médicos Indígenas, 1990.), donde el tema principal es la legislación de la medicina indígena tradicional. 
Para efectos de presentación, dividiré los tópicos correspondientes al tema, de la siguiente manera: En primer término, esbozaré los diferentes planteamientos y estrategias de diversos sectores del estado mexicano y chiapaneco en relación con la medicina indígena tradicional. Concretamente sobre el Capítulo IV de la Ley de Salud del Estado de Chiapas, donde abordaré lo referente al control y occidentalización de la medicina indígena tradicional y, las estrategias del Gobierno Federal, mismas que se ha pretendido implementar a través del Consejo Nacional de Médicos Indígenas Tradicionales (CONAMIT), instancia que ha sido instrumentada a través del Instituto Nacional Indigenista (INI), dependiente del Poder Ejecutivo Federal. Luego hablaré sobre las posiciones y propuestas de las organizaciones de médicos indígenas tradicionales del estado de Chiapas, particularmente sobre la manera cómo se concibe, entre las comunidades indígenas y sus médicos tradicionales, el proceso de legislación de la medicina indígena tradicional, a partir del impacto causado por el Capítulo IV de la Ley de Salud del Estado de Chiapas; las propuestas que ellos hacen para el proceso de legislación en materia de medicina indígena tradicional y, dentro de estas últimas, cómo visualizan la interrelación entre la medicina moderna y la medicina indígena tradicional dentro de la estrategia que ellos plantean para zonas indígenas.

\section{METODOS UTILIZADOS}

Partimos del principio de que la investigación, además de ser proyecto comunitario, en el sentido de responder a demandas y necesidades propias, y de hacer participar a los sujetos en el proceso de ejecución, debe, en la medida de lo posible, aportar beneficios inmediatos a la comunidad: Debido a lo cual este proyecto en particular se estructura a partir de la realización de dos talleres a realizarse en treintitrés comunidades de cinco municipios de los Altos de Chiapas; encaminándose, el primero, a la documentación de las características que tiene la tradición médica indígena en la actualidad, y el segundo a proporcionar capacitación jurídica, y sobre la base de ésta, generar un proceso de discusión y propuestas en torno a la legislación en materia de medicina indígena tradicional. La otra parte de la metodología está referida a la obligada documentación de lo ya escrito.

El producto de los aportes y discusiones resultado de los talleres son captados en audio cintas, para luego ser traducidos y vaciados en una base de datos computarizada. A partir de dicho material se elaboran memorias de los talleres, folletos dirigidos a la comunidad, procesamiento y análisis de la información obtenida, con los cuales se harán un informe final, varias publicaciones y presentaciones en los foros que sean factibles y pertinentes.

\section{DISCUSION}

Antes de adentrarme en la temática, quiero aclarar algunos aspectos conceptuales.

En la actualidad, algunos sectores categorizan dentro del ámbito de la medicina tradicional a todas aquellas prácticas que no forman parte de la medicina hegemónica, como: la Medicina indígena, la Galénica, la Homeopatía, la Acupuntura, la Ayurveda, la Naturoterapia, la Santería, la medicina invisible, etcétera. Si bien, algunas de éstas podrían considerarse como prácticas tradicionales y, de hecho, son hegemónicas dentro de sus propios contextos de origen, de lo cual los ejemplos más representativos son la medicina Ayurvédica, en la India, y la Acupuntura, dentro de la medicina China, otras son híbridos que han surgido a partir de la mezcla de varias de éstas. A este conjunto, dentro de nuestro contexto regional, las denominamos medicinas alternativas o paralelas, dentro de las que, aquí sí, podría estar incluida la medicina indígena tradicional.

El concepto de medicina tradicional, lo referimos, entonces, a aquellas prácticas médicas que han tenido su origen en el país o en la región, y que surgieron antes de la colonia, y/o bien que son inherentes a los orígenes de algún pueblo o grupo, lo cual de ninguna manera significa que no se hayan 
refuncionalizado infinidad de veces, incorporando elementos conceptuales, médicos y terapéuticos de las diferentes sociedades que los han dominado.

Por último, dado que no existe consenso respecto a los términos y sobre la base del acuerdo tomado por las organizaciones que integran el Consejo Nacional de Médicos Indígenas Tradicionales de México, en lo sucesivo me referiré a la Práctica Médica indígena como medicina indígena tradicional, con objeto de distinguirla de las otras. Hecha la salvedad, procedo.

Hablar a favor de la medicina indígena tradicional en un medio no antropológico y específicamente dentro del sector salud representa, en general, un verdadero problema, pero a la vez se ha convertido en algo necesario. Problema porque dicho sector, cumpliendo con su papel histórico e ideológico en la imposición de ideología y saberes entre los grupos subordinados, ha rechazado y descalificado con verdadero ahínco la práctica médica indígena tradicional. Necesario, primero, debido a que cada vez suena más fuertemente, en México, la reivindicación de la autonomía por parte de los grupos indígenas, pero no como una alternativa aislacionista sino en un sentido interactivo con la sociedad global, en donde las medicinas, cómo proponen los mismos médicos tradicionales, se encuentran para actuar coordinadamente en beneficio de la comunidad, borrando los estigmas de descalificación y persecución. Por otra parte, dentro del proceso de legislación del derecho de los pueblos indios, a partir del reconocimiento que de estos se hace en el Articulo Cuarto de la Constitución Mexicana (Salinas, 1990), y que, entre otras cosas, se ha concretado en un énfasis especial dirigido a la legislación de la medicina indígena tradicional, como parte importante de las políticas de salud para zonas indígenas dentro del marco del, llamado, nuevo orden económico. Política que establece la concomitante interrelación entre ésta y la medicina dominante, siendo precisamente el Sector Salud el que tendrá que entenderse con este mundo desconocido, aparentemente mágico y, para muchos, de charlatanería, una vez que se haya legislado al respecto. Hecho que ya se puede observar en los programas de salud de algunas instituciones de los Altos de Chiapas.

Si bien la medicina indígena tradicional siempre ha sido un tema de interés en el ámbito de la antropología, a principios de la década de los ochenta comienza a ventilarse, dentro de círculos oficiales, su inclusión dentro de las estrategias de atención a los grupos indígenas. A partir de entonces ha aumentado su importancia en nuestro país, en especial en los estados de la República que se caracterizan por tener un porcentaje elevado de población indígena, como es el caso de Chiapas, Oaxaca, Guerrero, Michoacán etc.

La inclusión de la medicina indígena tradicional como parte de las estrategias de atención en el estado de Chiapas se empieza a gestar paralelamente a la crisis que se suscitó en los programas de atención médica en áreas rurales, específicamente IMSS-COPLAMAR, que, a partir de la caída del precio del petróleo, a mediados de la década de los ochenta, redujo alarmantemente su cobertura y calidad de la atención, especialmente en las zonas más marginales y hecha a andar un programa nacional de medicina tradicional y herbolaria; medidas que se vieron reforzadas debido a la falta y encarecimiento de medicamentos.

El ahondamiento de la crisis y los cambios suscitados en la política económica a nivel mundial generaron, a su vez, mayor disminución del gasto en materia de salud, sobre todo el dirigido a grupos marginales, y el ajuste de las políticas de salud al nuevo orden económico, contemplándose, a mediano plazo, más recortes al gasto en salud de zonas marginales y el retiro parcial de las instituciones; depositando así, en la comunidad, la responsabilidad de la atención de los problemas de salud, tendencia que se confirma en la Ley de Salud (López Arellano \& Blanco Gil, 1993), reglamentaria del agregado que se hizo al Art. $4^{\circ}$ Constitucional, donde se plantea el Derecho a la protección de la salud. Y que se está concretando a través de las estrategias de Atención Primaria de Salud, que, como sabemos, contemplan acciones puntuales de 
prevención y atención y la participación de la comunidad, con todos sus recursos, en el cuidado y promoción de la salud, así como en la curación de sus enfermedades; entendiéndose por recursos propios no sólo aquellos representados por infraestructura, materiales, materia prima y mano de obra, sino también el involucramiento de sus prácticas médicas tradicionales o populares. Dentro de esta estrategia, también se considera la incorporación, al modelo médico hegemónico, de aquellas prácticas y principios de las medicinas alternativas, incluyendo la tradicional indígena, que a juicio de esta perspectiva tienen efectividad en la curación de enfermedades.

Así pues, la política neoliberal encaminada al abaratamiento de los costos de la atención de salud dirigida a los grupos marginales da su reconocimiento a la estructura médica indígena tradicional y la contempla dentro de su estrategia como instancia de primer contacto; reforzamiento que se espera se traduzca en un incremento de su consumo, con la consecuente absorción de ese gasto por parte de la comunidad, coadyuvando, de esta manera, a disminuir el volumen de enfermos atendidos por las instancias oficiales. Además de pretenderse el apoyo de los practicantes tradicionales para la instrumentación de algunos de los programas de prevención y fomento de la salud dirigidos a esta población. Por lo mismo, la legislación en materia de medicina tradicional es central dentro de esta estrategia y también en la situación y perspectivas futuras de la medicina indígena tradicional.

La situación antes descrita no puede transcurrir sin que el Estado pretenda mantener control sobre los médicos tradicionales indígenas, para lo cual establece la estrategia de legislar y normar las características que, además de lo correspondiente a la práctica tradicional indígena, pretende tenga su práctica médica global, es decir, la incorporación, dentro de su hacer, de los programas de salud que el Estado implemente - fomento a la salud, actividades elementales de primeros auxilios y atención médica simplificada (Congreso del Estado de Chiapas, Artículo 85, Capítulo IV Ley de salud p. 59) - con objeto de ir, gradualmente, medicalizando sus saberes, sin poder evitar el eterno afán descalificador e integracionista.

Los antecedentes del proceso de legislación en materia de derechos culturales de los pueblos indios, y específicamente en torno a la medicina tradicional, se encuentran, aparentemente, en las presiones ejercidas por la comunidad internacional, específicamente a través de sus agencias - Organización de las Naciones Unidas (ONU), Organización de las Naciones Unidas para la Agricultura y la Alimentación (FAO), Organización Internacional del Trabajo (OIT), Organización Mundial de la Salud (OMS), Organización Panamericana de la Salud (OPS) etc. -, dirigidas a los países con población indígena y que se han concretado en la firma de convenios, de los cuales el más importante es el Convenio 169 de la Organización Internacional del Trabajo (OIT, 1989). Este organismo, tomando en cuenta y "reconociendo las aspiraciones de los pueblos indígenas y tribales a asumir el control de sus propias instituciones, formas de vida, desarrollo económico, mantenimiento y fortalecimiento de sus identidades, lugares y religiones dentro del marco de los Estados en que viven", estableció este Convenio con la colaboración de diferentes organismos internacionales así como de la ONU. Cabe hacer notar que México se encuentra entre los países que lo suscribieron.

En 1990 el Lic. Carlos Salinas de Gortari, Presidente de la República, hace la propuesta de adición de un párrafo al artículo Cuarto Constitucional, para el reconocimiento de los derechos culturales de los pueblos indígenas (Salinas, 1990), misma que fue presentada al Congreso de la Unión, el 7 de Diciembre de 1990 y adicionado en enero de 1992. Los dos elementos fundamentales de este párrafo son: primero, que se reconoce la composición pluricultural de la nación, por lo tanto se "... protege el derecho a la diferencia dentro del marco de la convivencia y, segundo, prevee los instrumentos adecuados para proteger y desarrollar las culturas, organizaciones y recursos que sustentan a los pueblos indígenas de México" (Salinas, 1990: vii). 
El 30 de octubre, también de 1990, más de un año antes de la promulgación de la adición al Cuarto Constitucional, fue publicado en el Periódico Oficial No. 101 del Estado de Chiapas, El Capítulo IV, "De los Auxiliares de Fomento a la Salud", en la ley de Salud del Estado de Chiapas. Dicha ley se refiere básicamente a la situación legal a la que quedan sujetos los médicos indígenas tradicionales Chiapanecos, dentro del contexto del Sistema Estatal de Atención a la Salud. Para efectos de su elaboración, los médicos tradicionales, así como grupos indígenas representativos en el Estado de Chiapas, no fueron consultados.

El resultado es un documento que se compone de 10 artículos que, en su mayoría, son violatorios de lo recomendado en el Convenio 169 de la OIT, y aun de los conceptos básicos planteados en la adición al Cuarto Constitucional: "La nación mexicana tiene una composición pluricultural sustentada originalmente en sus pueblos indígenas. La ley protegerá y promoverá el desarrollo de sus lenguas, culturas, usos, costumbres, recursos y formas específicas de organización social..." (Salinas, 1990: vii). En un análisis efectuado sobre dicha ley en relación con los dos documentos señalados, se hicieron algunas de las siguientes observaciones (Page, 1992).

Desde el título del capítulo, se pretende ubicar, a los médicos indígenas tradicionales, en el escalón más bajo que puede existir dentro del escalafón de la "jerarquía blanca" al catalogarlos como " (...) auxiliares de fomento a la salud" (Congreso del Estado de Chiapas, 1990: 59). Lo que deja ver, por una parte, el gran desconocimiento que aún existe en los círculos legislativos y sanitarios del carácter de las medicinas indígenas tradicionales, como si pudieran ser incorporadas a los sistemas y organigramas del sector salud tal cual, sin tomar en consideración que se fundan en cosmovisiones distintas y que se ejercen, de hecho, en espacios de realidad que nos son ajenos.

En el artículo 81, primero de dicho capítulo, se plantea como objetivo central de la ley, “(...) el control del ejercicio de la medicina tradicional; en sus modalidades de actividad empírica y medicina indígena y herbolaria" (Congreso del Estado de Chiapas 1990: 59), lo que establece en forma precisa la intensión del estado de controlar e incidir en una práctica a la que solo-había perseguido y descalificado, pretendiendo con ello, además, invadir y determinar un espacio que había sido de injerencia exclusiva de los grupos indígenas.

El control se instrumenta, como se señala más adelante en el Artículo 85, inciso II, a través de la certificación por parte de la secretaria, del “(...) reconocimiento a las personas que acrediten los siguientes requisitos: Haber recibido adiestramiento suficiente en materia de fomento a la salud o en actividades elementales de primeros auxilios y atención médica simplificada en cualquier institución del sector salud. Previa acreditación con constancia expedida por la institución de salud que lo haya expedido" (Congreso del Estado de Chiapas, 1990: 59). Para luego asentar, en el Artículo 89 (Congreso del Estado de Chiapas, 1990: 60), que: "En ningún caso los reconocimientos a que se refiere el Artículo 85 (...) tendrán efecto de grado, ni certificarán validez de instrucción formal alguna, y solamente acreditarán tener calidad de auxiliares de fomento a la salud por lo tanto, el reconocimiento será individualmente y no a grupos ni organizaciones, como tampoco se otorgarán registros ni apoyarán trámites”.

La existencia de este inciso es también muestra del desconocimiento, por parte del Estado, de los procedimientos de fomento a la salud, preventivos y terapéuticos, que son parte de la medicina tradicional y confirma el grado de descalificación prejuiciada a que es sometida, al no validarle, a sus practicantes, destreza alguna; como si las comunidades indígenas no hubieran sobrevivido, a pesar de lo paupérrimo de su vida y del embate de la sociedad colonial, apoyados en forma determinante por su medicina tradicional.

Por otra parte, en dicho capítulo no se plantea la participación de los terapeutas ni en el diseño, ni en la organización e 
instrumentación de las capacitaciones; mucho menos, su planificación dentro del marco de sus culturas y en función de las necesidades e intereses de los afectados, como lo recomienda el Artículo 27, inciso 1, del Convenio 169 (OIT, 1989).

Con la aplicación de estos dos artículos, es decir la imposición de los cursos de fomento a la salud, primeros auxilios y atención médica simplificada, se generaría la medicalización de la práctica médica tradicional, con la clara intensión de hacer desaparecer gradualmente las medicinas indígenas tradicionales, lo que coadyuvaría a destruir un recurso, que con mucho es superior al servicio que pueden ofrecer los promotores de salud y los médicos pasantes que laboran con escasos recursos materiales en las regiones indígenas.

Una evaluación realizada en 1986 a personal auxiliar de salud y enfermeros indígenas del Instituto Nacional Indigenista (INI) en los Altos de Chiapas mostró que dicho personal, al no tener un sistema de seguimiento y capacitación adecuados, se volvió dependiente de esta técnica terapéutica. Por ejemplo, existen informes de la aplicación, en 20 días, de 171 inyecciones de medicamentos de uso restringido y peligroso; evidenciando una franca tendencia al abuso de este recurso que, utilizado inadecuadamente, se puede volver potencialmente iatrogénico.

Así mismo lo expresado por el Artículo 89 deja ver el desconocimiento que se tiene acerca de las formas de organización propias de los grupos indígenas, en el cual se da reconocimiento al manejo de las técnicas aprendidas en las capacitaciones mencionadas, dejando totalmente de lado lo relacionado con la medicina tradicional y desconociendo, además, las formas de organización propias de las comunidades y grupos indígenas, violando a su vez el derecho humano y constitucional (Salinas, 1990) a la reunión y organización en función de intereses comunes; y explícitamente contradice este planteamiento al no reconocer “... a grupos ni organizaciones, ..." que los grupos indígenas hayan creado para la defensa de sus costumbres, en este caso la organización para la defensa de su medicina tradicional.
También en el Artículo 85, inciso IV, que a la letra dice que los practicantes tradicionales no deben de: “(...) ser ministros de algún culto religioso o practicante sindicado en conflictos religiosos" (Congreso del Estado de Chiapas, 1990: 59), en donde también encontramos una relación de ignorancia, por parte de la Ley de Salud de Chiapas, hacia la práctica médica tradicional, ya que no se toma en consideración que un curandero prestigioso frecuentemente funge como autoridad religiosa entre su gente y que, como lo indica la tradición, no puede negarse a curar si se le solicita.

Por último e inevitablemente el postre coercitivo expresado en el Artículo 91 que, obviamente, dice: "Toda persona dedicada habitualmente a la actividad de fomento a la salud en forma empírica que no se ajuste a los lineamientos del presente capítulo no será reconocida como auxiliar de fomento a la salud por las autoridades sanitarias del Estado y, en su caso, se hará acreedora alas sanciones que determinen las leyes" (Congreso del Estado de Chiapas, 1990: 60) y que contradice totalmente el espíritu del Artículo 3, inciso 2 (OIT, 1989: 13) del Convenio, que dice: "No deberá emplearse ninguna forma de fuerza o de coerción que viole los derechos humanos y las libertades fundamentales de los pueblos interesados(...)”. Esta situación, en vez de “(...) promover y proteger el desarrollo de sus... costumbres...” (Salinas, 1990: vii, está supeditando y limitando este desarrollo a normas burocrático-administrativas.

En relación con el nivel federal, la instancia encargada de todo este proceso ha sido el INI, institución que se ha caracterizado por su mesura y discreción en este proceso, promoviendo y financiando, por un lado, investigaciones y consulta a organizaciones de médicos indígenas tradicionales y, por otro, la realización de varios congresos, que han tenido por resultado la conformación del Consejo Nacional de Médicos Indígenas Tradicionales (CONAMIT). Dentro del seno del consejo existe una comisión de legislación, encabezada por la OMIECH, que, 
hasta la fecha, no ha hecho un pronunciamiento definitivo; el único que existe es el que han hecho las organizaciones chiapanecas a través de este proyecto, y cuyos puntos de vista se exponen más adelante. En los foros mencionados, las organizaciones de médicos tradicionales indígenas de todo el país han denunciado, en repetidas ocasiones, la persecución y la falta de respeto, por parte de algunas instituciones y grupos sociales, a sus prácticas médicas, demandando que se legisle al respecto, demandas que son sistematizadas, por primera vez, en el "Primer encuentro de la Organización de Médicos Indígenas del Estado de Chiapas", que se realizó en San Cristóbal de las Casas, Chiapas, en 1986. Que se corrobora en el "Primer Congreso Nacional de Médicos Indígenas de México", que se realizó en Oaxtepec Mor., en 1989; en el "II Congreso Nacional de Médicos Indígenas de México", realizado en Pátzcuaro, Mich., en 1990, y en el "Segundo Congreso Nacional de Médicos Tradicionales Indígenas" y "Primer Encuentro Continental de Médicos Tradicionales Indígenas", que se realizaron en la ciudad de México, en agosto de 1992. Durante la segunda mitad de 1993, se realizó el taller de capacitación jurídica y opiniones y propuestas, cuyos resultados fueron presentados en una Asamblea de representantes, formulándose la siguiente propuesta, misma que ya ha sido presentada en varios foros con legisladores tanto del estado de Chiapas, como federales:

1. Toda legislación que se haga en relación con los pueblos indios será contenida en una Ley General de Derecho de los pueblos indios.

En este sentido, cabe el siguiente comentario:

Las características que adquirió el capitulo Cuarto de la Ley de Salud del estado de Chiapas es un ejemplo de legislación que nos permite ver que no es posible la incorporación de los derechos de los pueblos indios a los diferentes capítulos de las legislaciones estatales y federales, ya que obliga a que los artículos derivados se sometan a la estructura y objetivo general del capítulo correspondiente, adquiriendo, la ley, un carácter integracionista obligatorio y, por ende, de desintegración y destrucción de las culturas indígenas. Es por eso que consideramos que en las diferentes legislaciones, en caso de que éstas fueran imprescindibles y necesarias para los grupos indígenas, deben incluirse leyes generales que traten exclusivamente y en forma global el Derecho de los pueblos indios, sin contravenir ni los postulados de la constitución, ni la estructura de los diferentes códigos legales.

2. Que, en todo proceso legislativo en materia de derecho de los pueblos indios, realizado en los estados, sean tomados como directriz, la ley reglamentaria correspondiente al primer párrafo del Artículo $4^{\circ}$ constitucional y el Convenio No. 169 de la OIT, suscrito por México, y que constituyen los dos órganos máximos de referencia.

3. Que no es la medicina tradicional indígena, en sus mecanismos internos, lo legislable, dado que, al ser parte de la costumbre y la tradición de los pueblos indios, es improcedente, debido a que, como se precisa en el primer párrafo del Artículo $4^{\circ}$ Constitucional, "La Ley protegerá y promoverá el desarrollo de sus lenguas, culturas, usos, costumbres, recursos y formas específicas de organización social..." (Salinas, 1990: vi). Lo que, en todo caso, derivaría en la elaboración de un capítulo en las leyes de salud tanto federal como de los estados, en donde se establezca el respeto a los mecanismos propios, fincados en la costumbre indígena para la validación de la práctica de los médicos indígenas tradicionales, mismos que han sido aplicados durante siglos; además, se deben de asegurar los mecanismos jurídicos y financieros para su fortalecimiento y desarrollo; y que toda legislación que se realice, en torno a la medicina tradicional, tome como base la determinación de los médicos 
indígenas tradicionales de continuar siendo independientes, al mismo tiempo que estar coordinados con las instituciones de salud, apoyados por ellas pero no como asalariados o con menosprecio, sino, por el contrario, con respeto y apoyo mutuo.

Otro criterio que apoya la imposibilidad de legislar la práctica médica indígena está relacionado con el hecho de que a la fecha no existen parámetros ni criterios que permitan hacer una evaluación coherente, ni referida a la visión del mundo, ni respecto a las técnicas y procedimientos terapéuticos correspondientes a dichos sistemas médicos, así como su efectividad e impacto, para normar nuestra práctica.

Consideramos, en síntesis, que en todo caso lo legislable y a lo que habría que dar mayor importancia son las formas en que habrá de darse la interacción entre el Sector Salud y la Medicina Tradicional Indígena. En dicho proceso, el ejecutivo y los legisladores deben ser visionarios de la factibilidad del éxito o fracaso de esta relación operativa, la cual dependerá en buena medida de su proceder legislativo.

Así, en torno al fortalecimiento y lo legislable, en diferentes foros, hemos hecho las siguientes puntualizaciones y propuestas: Primero, que al respecto existen diferentes posiciones y propuestas, pudiendo aglutinarse todas en dos antagónicas. Una, que plantea la incorporación de la medicina tradicional a los esquemas jerárquicos de los sistemas nacionales y regionales de salud, en general subordinando a los médicos tradicionales mediante su ubicación en los niveles inferiores de los escalafones, en general, como promotores de salud o auxiliares de fomento a la salud, como se puede observar en el, ya mencionado, Capítulo 4ํㅜ de la Ley de Salud del Estado de Chiapas.

La otra posición, que es finalmente la que aglutina nuestra propuesta, así como de la mayoría de los terapeutas indígenas de los Altos de Chiapas, y que además es la recomendada tanto en círculos académicos abocados al estudio del problema, como en aquellos que han obtenido resultados óptimos al instrumentarlas, como sería el caso dentro de nuestro país de los "Programas de Medicina Mixta" que se llevan a cabo, desde hace algunos años, por el Instituto Nacional Indigenista/Instituto Nacional de la Nutrición Salvador Zubirán (INI/INNSZ), en diferentes partes del país y especialmente en la Sierra Norte de Puebla, para población Nahuatl y Totonaca, y en Nayarit para población Cora. Pues bien, esta estrategia plantea la coordinación entre ambas medicinas, en un plano de igualdad y mutuo respeto, que opere sobre la base de una estructura de referencia y contrarreferencia de pacientes, así como el trabajo conjunto en casos que así lo requieran.

En este sentido ha sido ampliamente estudiado y constatado, a nivel continental, que los marcos conceptuales de las medicinas tradicionales indígenas, en relación con el de la medicina moderna, son diferentes y están referidos a percepciones y concepciones del mundo también muy diferentes, tales que no permiten compatibilidad alguna. Sin embargo, ante la prioridad de dicha funcionalidad, proponemos que la legislación se haga sobre la base de dicha coordinación, estableciéndose sobre la base de los límites y similitudes nosológicas que existan entre ambas medicinas, es decir, determinar en que nosologías hay compatibilidad y viabilidad terapéutica mixta, independientemente de la interpretación del origen del problema, y en ellas estudiar la eficacia de las terapéuticas de ambas medicinas, lo que daría el primer criterio de canalización y trabajo conjunto y, por otro lado, establecer las nosologías que sólo existen para una u otra medicina, es decir, lo que marca el límite y la diferencia, lo que nos daría el segundo criterio de canalización.

4. Queremos que el trabajo con el Sector Salud tenga como base un programa especial hecho por nosotros y las instituciones; que contenga apoyos materiales y financieros para las diferentes ramas de la medicina indígena tradicional.

5. Que el Gobierno Estatal apoye, a través de sus instituciones proyectos 
de: investigación, capacitación y servicio, abocados a afinar las relaciones entre medicina tradicional y los sistemas de salud.

6. Consideramos que en los planes de estudio de las instituciones formadoras de recursos humanos para la salud, sobre todo de aquellos que realizarán su trabajo profesional en dichas zonas, debe ser considerada la inclusión de materias como antropología médica, ya que es necesario que sus egresados tengan algún conocimiento de lo que encontrarán al venir a nuestras comunidades.

7. La adecuación de los servicios, en clínicas y hospitales de las regiones indias, a las necesidades de estas regiones; adecuación que tendría que realizarse en coordinación con los médicos tradicionales y representantes de las comunidades.

8. La participación de las comunidades indígenas y médicos indígenas en la planificación, la programación, el desarrollo y la evaluación de los programas oficiales de salud, aprovechando los conocimientos que tenemos de las regiones, de las necesidades y de la penetración dentro de nuestras comunidades.

9. Que el Gobierno del Estado apoye la documentación, así como la identificación y la preservación de los recursos naturales usados por la Medicina Indígena Tradicional.

10. La libre participación del médico tradicional como autoridad religiosa, dentro de la organización jerárquica tradicional.

11. Respetar los mecanismos propios, de la costumbre, mediante los cuales se reconoce cuando una persona es médico indígena tradicional en su comunidad y se acepta su trabajo, y cuando es charlatán y se le rechaza.
12. No se podrá obligar a los médicos tradicionales a tomar cursos con contenidos propios de la medicina oficial, como de fomento a la salud, primeros auxilios y atención médica simplificada. En su lugar se sugiere que se fijen mecanismos encaminados a la organización de encuentros entre médicos tradicionales y la creación de Centros de Desarrollo de la Medicina Tradicional, que tengan por objeto el intercambio, la enseñanza y el fortalecimiento de las técnicas y procedimientos de nuestra medicina.

13. Se propone que se fijen mecanismos legales que permitan el apoyo a organizaciones de médicos indígenas tradicionales encaminadas a la conservación, el rescate, el enriquecimiento y el fortalecimiento de la medicina tradicional.

Por otro lado, es necesario considerar que, dependiendo de las características que adquieran las futuras legislaciones en relación con la medicina tradicional, dependerá el éxito o fracaso de la estrategia particular de atención Primaria de Salud, que pretende la incorporación de los médicos tradicionales a los Sistemas de Salud, así como también de la el situación y el futuro de la medicina tradicional.

\section{RESUMEN}

\section{PAGE, J. T. P. Política Sanitaria y} Legislación en Materia de Medicina Indígeña Tradicional en México. Cad. Saúde Públ., Rio de Janeiro, 11 (2): 201-211, abr/jun, 1995.

Diferentes instancias de gobierno, tanto estatales como federales, están tratando de establecer el control y la medicalización de las medicinas tradicionales, y a través de su reconocimiento fortalecerla como instancia de primer contacto, generando, de esta manera, que la comunidad absorba los costos iniciales de la atención médica, lo que coadyuva a disminuir el volumen de enfermos atendidos 
por las instancias oficiales; obteniéndose así el abaratamiento de los costos de la atención de salud institucional dirigida a estos grupos. Los médicos indígenas tradicionales y sus organizaciones ven a esta ley cómo una navaja de doble filo, vislumbrando, por una parte, la posibilidad de contar con instrumentos legales que les protejan ante la sociedad global y, por otra, el peligro de ver su cultura .y sus prácticas destruidas, por lo que han optado por buscar asesoría, capacitación, así como por participar activamente en el proceso de construcción de estas leyes. El resultado de dicho proyecto es que han aprendido sobre Derecho Positivo Mexicano; sobre esta base, han podido discutir y concluir que es lo que quieren por ley, para lo que han elaborado una propuesta, incluida en este documento.

Palabras Clave: Salud Indígena; Política Sanitaria; Sistemas de Salud; Legislación en Salud; Medicina Indígena Tradicional

\section{REFERENCIAS BIBLIOGRAFICAS}

CONGRESO DEL ESTADO DE CHIAPAS, 1990. De los auxiliares de fomento a la salud. In: Ley de Salud. Recursos Humanos para la Salud, 30 de Octubre, Capítulo IV, pp. 59-61, Tuxtla Gtz., Chiapas: Estado de Chiapas, México.

CONGRESO NACIONAL DE MEDICOS INDIGENAS, 1989. Declaración de Oaxtepec sobre Medicina Indígena. Oaxtepec, Mor., México. 1990. Documento Pátzcuaro. Reconocimiento y Legalización de la Medicina Tradicional. México: Instituto Nacional Indigenista. (Mimeo.)

LOPEZ ARELLANO, O. \& BLANCO GIL, J., 1993. La Modernización Neoliberal en Salud (México en los Ochenta). México: Universidad Autónoma Metropolitana.

OIT (Organización Internacional del Trabajo), 1989. Sobre Pueblos Indígenas y Tribales en Países Independientes. Convenio № 169, pp. 01-26, OIT. (Mimeo.)

PAGE, J. P., 1992. Comentarios al Capítulo IV de la Ley de Salud del Estado de Chiapas, en torno a la Medicina Indígena Tradicional, pp. 01-15, México. (Mimeo.)

SALINAS, C. G. 1990. Artículo 4ํ de la Constitución Federal de la Rep. Mexicana, p. i-viii, México: Presidencia de la República. 